\title{
Quantitative Hydrogen/Deuterium Exchange Mass Spectrometry
}

Yoshitomo Hamuro ${ }^{1,2,3}$

${ }^{1}$ ExSAR Corporation, 11 Deer Park Drive, Suite 103, Monmouth Junction, NJ 08852

2 Current address: Janssen Research and Development, LLC, 1400 McKean Road, Spring House, PA 19477

${ }^{3}$ Corresponding author: Yoshitomo Hamuro; yhamuro@its.jnj.com

\section{Supporting Information}

Table of contents

1. Observed Backbone Amide Exchange Rates of Cytochrome c

2. Relative Intrinsic Backbone Amide Exchange Rates Depending on Sidechains

3. Derivations to Calculate Area between Two Exponential Curves

3.1. Calculation of Area between Two Exponential Curves by Frullani integral

3.2. Calculation of Area between Two Stretched Exponential Curves

3.3. Calculation of Protection Factor between Two Stretched Exponential Curves 


\section{Observed Backbone Amide Exchange Rates of Cytochrome c}

Table S1. Observed backbone amide exchange rates of Cytochrome c $\left(\mathrm{s}^{-1}\right) .{ }^{\mathrm{a}}$

\begin{tabular}{|c|c|c|c|c|c|c|c|}
\hline$\#$ & AA & $\mathrm{NMR}^{\mathrm{b}}$ & Pepsin $^{c}$ & Pepsin/FPXIII ${ }^{d}$ & ETD $^{\mathrm{e}}$ & Isotope $^{c}$ & Combined \\
\hline 1 & $\mathrm{G}$ & $\begin{array}{l}---- \\
--1\end{array}$ & ---- & $\begin{array}{l}---- \\
--1\end{array}$ & $\begin{array}{ll}---- \\
--1\end{array}$ & $2.6 \mathrm{E}+00$ & $2.6 \mathrm{E}+00$ \\
\hline 2 & $\mathrm{D}$ & ----- & ----- & ----- & ----- & $1.3 \mathrm{E}-01$ & $1.3 \mathrm{E}-01$ \\
\hline 3 & $\mathrm{~V}$ & ----- & ----- & ----- & $1.6 \mathrm{E}-01$ & $8.4 \mathrm{E}-02$ & $8.4 \mathrm{E}-02$ \\
\hline 4 & $\mathrm{E}$ & ----- & ----- & ----- & 4.9E-03 & 7.3E-02 & 7.3E-02 \\
\hline 5 & $\mathrm{~K}$ & ----- & ----- & ----- & $2.3 \mathrm{E}-03$ & $2.1 \mathrm{E}-02$ & $2.1 \mathrm{E}-02$ \\
\hline 6 & $\mathrm{G}$ & ----- & ----- & ----- & ----- & $6.6 \mathrm{E}-07$ & $6.6 \mathrm{E}-07$ \\
\hline 7 & K & $2.6 \mathrm{E}-04$ & ----- & ----- & ----- & 4.2E-04 & $2.6 \mathrm{E}-04$ \\
\hline 8 & $\mathrm{~K}$ & 7.9E-04 & ----- & $6.2 \mathrm{E}-05$ & ----- & $2.2 \mathrm{E}-03$ & 7.9E-04 \\
\hline 9 & I & $3.2 \mathrm{E}-05$ & ----- & $1.4 \mathrm{E}-05$ & ----- & $4.4 \mathrm{E}-05$ & $3.2 \mathrm{E}-05$ \\
\hline 10 & $\mathrm{~F}$ & $5.7 \mathrm{E}-08$ & ----- & $1.0 \mathrm{E}-06$ & ----- & $9.4 \mathrm{E}-09$ & $5.7 \mathrm{E}-08$ \\
\hline 11 & $\mathrm{~V}$ & $1.6 \mathrm{E}-05$ & ----- & $1.3 \mathrm{E}-05$ & ----- & $1.2 \mathrm{E}-06$ & $1.6 \mathrm{E}-05$ \\
\hline 12 & Q & $2.1 \mathrm{E}-04$ & ----- & $8.4 \mathrm{E}-05$ & ----- & $1.9 \mathrm{E}-04$ & $2.1 \mathrm{E}-04$ \\
\hline 13 & $\mathrm{~K}$ & 4.9E-05 & ----- & $1.5 \mathrm{E}-05$ & ----- & $2.3 \mathrm{E}-05$ & $4.9 \mathrm{E}-05$ \\
\hline 14 & $\mathrm{C}$ & $7.6 \mathrm{E}-05$ & ----- & ----- & ----- & $2.3 \mathrm{E}-05$ & $7.6 \mathrm{E}-05$ \\
\hline 15 & A & 4.7E-04 & ----- & ----- & ----- & $1.4 \mathrm{E}-03$ & 4.7E-04 \\
\hline 16 & $\mathrm{Q}$ & & ----- & ----- & ----- & $1.6 \mathrm{E}+00$ & $1.6 \mathrm{E}+00$ \\
\hline 17 & $\mathrm{C}$ & & ----- & ----- & ----- & $3.3 \mathrm{E}-01$ & $3.3 \mathrm{E}-01$ \\
\hline 18 & $\mathrm{H}$ & 4.3E-04 & ----- & ----- & ----- & $1.9 \mathrm{E}-04$ & 4.3E-04 \\
\hline 19 & $\mathrm{~T}$ & $3.6 \mathrm{E}-04$ & ----- & ----- & ----- & $1.9 \mathrm{E}-04$ & $3.6 \mathrm{E}-04$ \\
\hline 20 & $\mathrm{~V}$ & ----- & ----- & ----- & ----- & $1.2 \mathrm{E}+01$ & $1.2 \mathrm{E}+01$ \\
\hline 21 & $\mathrm{E}$ & ----- & ----- & $2.9 \mathrm{E}+02$ & ----- & $6.2 \mathrm{E}+01$ & $2.9 \mathrm{E}+02$ \\
\hline 22 & $\mathrm{~K}$ & ----- & ----- & $4.0 \mathrm{E}-01$ & ----- & ----- & $4.0 \mathrm{E}-01$ \\
\hline 23 & $\mathrm{G}$ & ----- & ----- & $1.0 \mathrm{E}-06$ & ----- & ----- & $1.0 \mathrm{E}-06$ \\
\hline 24 & $\mathrm{G}$ & ----- & ----- & ----- & ----- & $9.9 \mathrm{E}+02$ & $9.9 \mathrm{E}+02$ \\
\hline 25 & $\mathrm{~K}$ & ----- & ----- & ----- & ----- & $3.8 \mathrm{E}-02$ & $3.8 \mathrm{E}-02$ \\
\hline 26 & $\mathrm{H}$ & ----- & ----- & ----- & ----- & $1.4 \mathrm{E}-04$ & $1.4 \mathrm{E}-04$ \\
\hline 27 & $\mathrm{~K}$ & ----- & ----- & ----- & ----- & $2.4 \mathrm{E}-07$ & $2.4 \mathrm{E}-07$ \\
\hline 28 & $\mathrm{~T}$ & ----- & ----- & ----- & ----- & $6.9 \mathrm{E}-07$ & $6.9 \mathrm{E}-07$ \\
\hline 29 & $\mathrm{G}$ & $1.1 \mathrm{E}-04$ & ----- & ----- & ----- & $1.3 \mathrm{E}-04$ & $1.1 \mathrm{E}-04$ \\
\hline 30 & $\mathrm{P}$ & & ----- & ----- & ----- & ----- & ----- \\
\hline 31 & $\mathrm{~N}$ & $2.2 \mathrm{E}-02$ & ----- & ----- & ----- & $3.8 \mathrm{E}-02$ & $2.2 \mathrm{E}-02$ \\
\hline 32 & $\mathrm{~L}$ & $4.0 \mathrm{E}-06$ & ----- & ----- & ----- & $3.2 \mathrm{E}-05$ & $4.0 \mathrm{E}-06$ \\
\hline 33 & $\mathrm{H}$ & $2.0 \mathrm{E}-05$ & ----- & $2.6 \mathrm{E}-06$ & ----- & $3.2 \mathrm{E}-05$ & $2.0 \mathrm{E}-05$ \\
\hline 34 & $\mathrm{G}$ & ----- & ----- & $1.0 \mathrm{E}-06$ & ----- & $2.4 \mathrm{E}-07$ & $1.0 \mathrm{E}-06$ \\
\hline 35 & $\mathrm{~L}$ & $3.9 \mathrm{E}-04$ & ----- & $1.0 \mathrm{E}-06$ & ----- & $1.3 \mathrm{E}-03$ & $3.9 \mathrm{E}-04$ \\
\hline 36 & $\mathrm{~F}$ & $8.4 \mathrm{E}-05$ & ----- & $1.5 \mathrm{E}-05$ & ----- & $1.3 \mathrm{E}-03$ & $8.4 \mathrm{E}-05$ \\
\hline 37 & $\mathrm{G}$ & $1.0 \mathrm{E}-03$ & ----- & $1.4 \mathrm{E}-04$ & ----- & $1.3 \mathrm{E}-03$ & $1.0 \mathrm{E}-03$ \\
\hline 38 & $\mathrm{R}$ & $1.5 \mathrm{E}-03$ & ----- & $9.4 \mathrm{E}-05$ & ----- & $1.3 \mathrm{E}-03$ & $1.5 \mathrm{E}-03$ \\
\hline 39 & $\mathrm{~K}$ & ----- & ----- & ----- & ----- & 4.7E-07 & 4.7E-07 \\
\hline 40 & $\mathrm{~T}$ & $3.9 \mathrm{E}-03$ & ----- & ----- & ----- & $3.7 \mathrm{E}-03$ & $3.9 \mathrm{E}-03$ \\
\hline
\end{tabular}




\begin{tabular}{|c|c|c|c|c|c|c|c|}
\hline 41 & $\mathrm{G}$ & ----- & ----- & ----- & $5.8 \mathrm{E}-03$ & $1.3 \mathrm{E}-02$ & $1.3 \mathrm{E}-02$ \\
\hline 42 & $\mathrm{Q}$ & $5.5 \mathrm{E}-03$ & ----- & ----- & ----- & $1.3 \mathrm{E}-02$ & $5.5 \mathrm{E}-03$ \\
\hline 43 & $\mathrm{~A}$ & $1.4 \mathrm{E}-02$ & ----- & ----- & ----- & $1.3 \mathrm{E}-02$ & $1.4 \mathrm{E}-02$ \\
\hline 44 & $\mathrm{P}$ & ----- & ----- & ----- & ----- & ----- & ----- \\
\hline 45 & $\mathrm{G}$ & ----- & ----- & ----- & $1.2 \mathrm{E}-02$ & 7.2E-02 & 7.2E-02 \\
\hline 46 & $\mathrm{~F}$ & ----- & ----- & $1.1 \mathrm{E}-02$ & $4.1 \mathrm{E}+02$ & $1.1 \mathrm{E}+02$ & $1.1 \mathrm{E}-02$ \\
\hline 47 & $\mathrm{~T}$ & ----- & $1.5 \mathrm{E}+02$ & $5.3 \mathrm{E}+01$ & ----- & $1.5 \mathrm{E}+02$ & $1.5 \mathrm{E}+02$ \\
\hline 48 & $\mathrm{Y}$ & ----- & ----- & $5.0 \mathrm{E}-01$ & ----- & ----- & $5.0 \mathrm{E}-01$ \\
\hline 49 & $\mathrm{~T}$ & $2.6 \mathrm{E}-02$ & 8.8E-03 & $6.1 \mathrm{E}-03$ & ----- & 8.8E-03 & $2.6 \mathrm{E}-02$ \\
\hline 50 & $\mathrm{D}$ & ----- & ----- & $1.5 \mathrm{E}+01$ & ----- & $1.5 \mathrm{E}+02$ & $1.5 \mathrm{E}+01$ \\
\hline 51 & $\mathrm{~A}$ & ----- & ----- & 4.0E-01 & ----- & $1.7 \mathrm{E}+01$ & 4.0E-01 \\
\hline 52 & $\mathrm{~N}$ & $9.1 \mathrm{E}-03$ & ----- & $3.7 \mathrm{E}-03$ & ----- & $1.2 \mathrm{E}-02$ & $9.1 \mathrm{E}-03$ \\
\hline 53 & $\mathrm{~K}$ & $1.3 \mathrm{E}-02$ & ----- & $3.6 \mathrm{E}-03$ & ----- & $2.0 \mathrm{E}-02$ & $1.3 \mathrm{E}-02$ \\
\hline 54 & $\mathrm{~N}$ & $2.1 \mathrm{E}-02$ & ----- & 4.0E-01 & ----- & $2.8 \mathrm{E}-02$ & $2.1 \mathrm{E}-02$ \\
\hline 55 & $\mathrm{~K}$ & ----- & ----- & $8.6 \mathrm{E}-03$ & ----- & $1.3 \mathrm{E}+00$ & 8.6E-03 \\
\hline 56 & $\mathrm{G}$ & ----- & ----- & 4.0E-01 & ----- & $1.3 \mathrm{E}+00$ & 4.0E-01 \\
\hline 57 & I & $3.3 \mathrm{E}-03$ & ----- & $3.8 \mathrm{E}-01$ & ----- & $2.1 \mathrm{E}-03$ & $3.3 \mathrm{E}-03$ \\
\hline 58 & $\mathrm{~T}$ & ----- & ----- & $2.0 \mathrm{E}-02$ & ----- & $4.0 \mathrm{E}-01$ & 2.0E-02 \\
\hline 59 & $\mathrm{~W}$ & $1.4 \mathrm{E}-04$ & ----- & $1.0 \mathrm{E}-06$ & $2.0 \mathrm{E}-04$ & $3.6 \mathrm{E}-04$ & $1.4 \mathrm{E}-04$ \\
\hline 60 & $\mathrm{~K}$ & $1.6 \mathrm{E}-05$ & ----- & $2.4 \mathrm{E}-06$ & $9.0 \mathrm{E}-05$ & 1.1E-04 & $1.6 \mathrm{E}-05$ \\
\hline 61 & $\mathrm{E}$ & ----- & ----- & $4.9 \mathrm{E}+02$ & $1.2 \mathrm{E}-01$ & $9.5 \mathrm{E}-02$ & $4.9 \mathrm{E}+02$ \\
\hline 62 & $\mathrm{E}$ & ----- & ----- & $2.2 \mathrm{E}-01$ & $3.1 \mathrm{E}+01$ & $3.2 \mathrm{E}-02$ & 2.2E-01 \\
\hline 63 & $\mathrm{~T}$ & ----- & ----- & 7.6E-03 & $4.8 \mathrm{E}-02$ & 9.4E-09 & 7.6E-03 \\
\hline 64 & $\mathrm{~L}$ & $1.5 \mathrm{E}-05$ & $3.6 \mathrm{E}-05$ & $6.2 \mathrm{E}-06$ & $1.0 \mathrm{E}-03$ & $3.6 \mathrm{E}-05$ & $1.5 \mathrm{E}-05$ \\
\hline 65 & $\mathrm{M}$ & $2.6 \mathrm{E}-06$ & $1.7 \mathrm{E}-05$ & $1.0 \mathrm{E}-06$ & ----- & $1.7 \mathrm{E}-05$ & $2.6 \mathrm{E}-06$ \\
\hline 66 & $\mathrm{E}$ & $5.8 \mathrm{E}-04$ & $1.6 \mathrm{E}-04$ & $4.0 \mathrm{E}-05$ & ----- & $1.6 \mathrm{E}-04$ & $5.8 \mathrm{E}-04$ \\
\hline 67 & $\mathrm{Y}$ & $3.3 \mathrm{E}-05$ & $2.3 \mathrm{E}-05$ & $2.4 \mathrm{E}-05$ & & $2.3 \mathrm{E}-05$ & $3.3 \mathrm{E}-05$ \\
\hline 68 & $\mathrm{~L}$ & $1.2 \mathrm{E}-08$ & $3.5 \mathrm{E}-06$ & $1.0 \mathrm{E}-06$ & ----- & $3.5 \mathrm{E}-06$ & $1.2 \mathrm{E}-08$ \\
\hline 69 & $\mathrm{E}$ & $9.4 \mathrm{E}-06$ & ----- & $1.3 \mathrm{E}-04$ & ----- & $6.6 \mathrm{E}-07$ & $9.4 \mathrm{E}-06$ \\
\hline 70 & $\mathrm{~N}$ & 7.4E-04 & ----- & $1.7 \mathrm{E}-04$ & $1.8 \mathrm{E}-02$ & 8.0E-04 & 7.4E-04 \\
\hline 71 & $\mathrm{P}$ & ----- & ----- & ----- & ----- & ----- & ----- \\
\hline 72 & $\mathrm{~K}$ & ----- & ----- & $7.5 \mathrm{E}-03$ & & $2.5 \mathrm{E}-02$ & 7.5E-03 \\
\hline 73 & $\mathrm{~K}$ & 7.1E-03 & ----- & 4.9E-05 & ----- & $9.8 \mathrm{E}-03$ & 7.1E-03 \\
\hline 74 & $\mathrm{Y}$ & $2.1 \mathrm{E}-04$ & ----- & 7.0E-04 & $1.7 \mathrm{E}-03$ & 8.0E-04 & $2.1 \mathrm{E}-04$ \\
\hline 75 & I & $1.0 \mathrm{E}-04$ & ----- & $3.6 \mathrm{E}-05$ & 7.3E-04 & $1.9 \mathrm{E}-04$ & $1.0 \mathrm{E}-04$ \\
\hline 76 & $\mathrm{P}$ & ----- & ----- & ----- & ----- & ----- & ----- \\
\hline 77 & $\mathrm{G}$ & ----- & ----- & $5.5 \mathrm{E}-04$ & $2.2 \mathrm{E}-04$ & 7.2E-02 & $5.5 \mathrm{E}-04$ \\
\hline 78 & $\mathrm{~T}$ & ----- & ----- & $3.5 \mathrm{E}+01$ & $6.1 \mathrm{E}-04$ & $1.0 \mathrm{E}+02$ & $3.5 \mathrm{E}+01$ \\
\hline 79 & $\mathrm{~K}$ & $2.1 \mathrm{E}-02$ & ----- & $1.1 \mathrm{E}-02$ & $2.3 \mathrm{E}-02$ & $2.5 \mathrm{E}-02$ & 2.1E-02 \\
\hline 80 & $\mathrm{M}$ & $3.1 \mathrm{E}-02$ & ----- & $3.0 \mathrm{E}-03$ & $1.2 \mathrm{E}-02$ & $2.5 \mathrm{E}-02$ & $3.1 \mathrm{E}-02$ \\
\hline 81 & I & ----- & 2.2E-01 & $1.6 \mathrm{E}-01$ & $6.8 \mathrm{E}-02$ & 2.2E-01 & 2.2E-01 \\
\hline 82 & $\mathrm{~F}$ & ----- & $3.1 \mathrm{E}+01$ & $2.4 \mathrm{E}+01$ & $2.0 \mathrm{E}+01$ & $3.1 \mathrm{E}+01$ & $3.1 \mathrm{E}+01$ \\
\hline 83 & $\mathrm{~A}$ & ----- & $9.4 \mathrm{E}+02$ & $6.1 \mathrm{E}+01$ & $1.1 \mathrm{E}+03$ & $9.4 \mathrm{E}+02$ & $9.4 \mathrm{E}+02$ \\
\hline 84 & $\mathrm{G}$ & ----- & 2.2E-01 & $9.1 \mathrm{E}-02$ & $3.7 \mathrm{E}-01$ & $2.2 \mathrm{E}-01$ & 2.2E-01 \\
\hline
\end{tabular}




\begin{tabular}{rrrrrrrr}
85 & $\mathrm{I}$ & $1.1 \mathrm{E}-03$ & ----- & $1.9 \mathrm{E}-04$ & $1.4 \mathrm{E}-03$ & $1.4 \mathrm{E}-03$ & $1.1 \mathrm{E}-03$ \\
86 & $\mathrm{~K}$ & ----- & ----- & $3.8 \mathrm{E}+01$ & $6.6 \mathrm{E}+01$ & $1.2 \mathrm{E}+02$ & $3.8 \mathrm{E}+01$ \\
87 & $\mathrm{~K}$ & ----- & ----- & $9.1 \mathrm{E}+01$ & ----- & $1.2 \mathrm{E}+02$ & $9.1 \mathrm{E}+01$ \\
88 & $\mathrm{~K}$ & ----- & ----- & $5.3 \mathrm{E}-01$ & ----- & $4.6 \mathrm{E}+00$ & $5.3 \mathrm{E}-01$ \\
89 & $\mathrm{~T}$ & ----- & ----- & $4.4 \mathrm{E}+00$ & ----- & $7.9 \mathrm{E}-01$ & $4.4 \mathrm{E}+00$ \\
90 & $\mathrm{E}$ & ----- & ----- & $1.3 \mathrm{E}-03$ & ----- & $1.7 \mathrm{E}-02$ & $1.3 \mathrm{E}-03$ \\
91 & $\mathrm{R}$ & $1.2 \mathrm{E}-05$ & ----- & $1.0 \mathrm{E}-06$ & ----- & $2.8 \mathrm{E}-05$ & $1.2 \mathrm{E}-05$ \\
92 & $\mathrm{E}$ & $6.6 \mathrm{E}-06$ & ----- & $1.0 \mathrm{E}-06$ & ----- & $5.9 \mathrm{E}-07$ & $6.6 \mathrm{E}-06$ \\
93 & $\mathrm{D}$ & $1.4 \mathrm{E}-05$ & ----- & $1.0 \mathrm{E}-06$ & ----- & $2.9 \mathrm{E}-05$ & $1.4 \mathrm{E}-05$ \\
94 & $\mathrm{~L}$ & $3.8 \mathrm{E}-08$ & ----- & $1.0 \mathrm{E}-06$ & ----- & $5.8 \mathrm{E}-07$ & $3.8 \mathrm{E}-08$ \\
95 & $\mathrm{I}$ & $3.8 \mathrm{E}-09$ & $9.4 \mathrm{E}-09$ & $1.0 \mathrm{E}-06$ & $7.1 \mathrm{E}-07$ & $9.4 \mathrm{E}-09$ & $3.8 \mathrm{E}-09$ \\
96 & $\mathrm{~A}$ & $3.7 \mathrm{E}-08$ & $5.3 \mathrm{E}-08$ & $1.0 \mathrm{E}-06$ & $1.4 \mathrm{E}-06$ & $5.3 \mathrm{E}-08$ & $3.7 \mathrm{E}-08$ \\
97 & $\mathrm{Y}$ & $2.5 \mathrm{E}-08$ & $1.1 \mathrm{E}-07$ & $1.0 \mathrm{E}-06$ & ----- & $1.1 \mathrm{E}-07$ & $2.5 \mathrm{E}-08$ \\
98 & $\mathrm{~L}$ & $3.8 \mathrm{E}-10$ & $9.5 \mathrm{E}-08$ & $1.0 \mathrm{E}-06$ & $5.3 \mathrm{E}-08$ & $9.5 \mathrm{E}-08$ & $3.8 \mathrm{E}-10$ \\
99 & $\mathrm{~K}$ & $1.2 \mathrm{E}-07$ & ----- & ----- & ----- & $9.4 \mathrm{E}-09$ & $1.2 \mathrm{E}-07$ \\
100 & $\mathrm{~K}$ & $2.5 \mathrm{E}-04$ & ----- & ----- & ----- & $4.9 \mathrm{E}-04$ & $2.5 \mathrm{E}-04$ \\
101 & $\mathrm{~A}$ & $4.3 \mathrm{E}-03$ & ----- & ----- & ----- & $2.2 \mathrm{E}-03$ & $4.3 \mathrm{E}-03$ \\
102 & $\mathrm{~T}$ & ----- & ----- & ----- & $4.2 \mathrm{E}-04$ & $8.7 \mathrm{E}-05$ & $8.7 \mathrm{E}-05$ \\
103 & $\mathrm{~N}$ & ----- & ----- & ----- & $3.1 \mathrm{E}-03$ & $2.2 \mathrm{E}-02$ & $2.2 \mathrm{E}-02$ \\
104 & $\mathrm{E}$ & ----- & ----- & ----- & $5.9 \mathrm{E}-02$ & $3.5 \mathrm{E}-01$ & $3.5 \mathrm{E}-01$ \\
\hline
\end{tabular}

a The first choice is HDX-NMR data. The second choice is HDX-MS data with pepsin digestion. The third choice is HDX-MS data with pepsin/FPXIII digestion. The fourth choice is ETDHDX-MS data. The fifth choice is HDX-MS data using isotope envelope.

b Milne, J.S., Mayne, L., Roder, H., Wand, A.J., Englander, S.W.: Determinants of protein hydrogen exchange studied in equine cytochrome c. Protein Science. 7, 739-745 (1998)

c Hamuro, Y.: Determination of equine Cytochrome c backbone amide hydrogen/deuterium exchange rates by mass spectrometry using a wider time window and isotope envelope. J. Am. Soc. Mass Spectrom. 28, 486-497 (2017)

d Hamuro, Y., Zhang, T.: High-Resolution HDX-MS of Cytochrome c Using Pepsin/Fungal Protease Type XIII Mixed Bed Column. J. Am. Soc. Mass Spectrom. 30, 227-234 (2019)

e Hamuro, Y, E, S.Y.: Determination of Backbone Amide Hydrogen Exchange Rates of Cytochrome c Using Partially Scrambled Electron Transfer Dissociation Data. J. Am. Soc. Mass Spectrom. 29, 989-1001 (2018) 


\section{Relative Intrinsic Backbone Amide Exchange Rates Depending on Sidechains}

Table S2. Relative HDX rates of $-\mathrm{X}-\mathrm{C}(=\mathrm{O})-\mathrm{NH}-\mathrm{Y}$ - at $\mathrm{p} D_{\text {corr }} 7.4$ and $23{ }^{\circ} \mathrm{C}\left(\mathrm{s}^{-1}\right)$ calculated by

HXrates2018_HD from Englander's lab to mimic HDX reaction compared with polyalanine. ${ }^{\mathrm{a}}$

\begin{tabular}{|c|c|c|c|c|c|c|c|c|c|c|c|c|c|c|c|c|c|c|c|c|c|}
\hline$\overline{X I Y}$ & $\mathrm{C}$ & $\mathrm{N}$ & $\mathrm{H}$ & $S$ & $\mathrm{D}$ & $\mathrm{R}$ & $\mathrm{Q}$ & A & $\mathrm{M}$ & $G$ & $\mathrm{~K}$ & $\mathrm{~T}$ & $\mathrm{E}$ & $\mathrm{F}$ & $\bar{Y}$ & $\mathrm{~W}$ & $\mathrm{~L}$ & V & 1 & $P$ & Ave. \\
\hline C & 14.8 & 11.0 & 9.0 & 8.3 & 4.5 & 4.2 & 4.1 & 3.5 & 3.5 & 3.3 & 3.2 & 3.0 & 2.8 & 2.1 & 1.9 & 1.4 & 0.9 & 0.7 & 0.7 & & 4.4 \\
\hline $\mathrm{H}$ & 12.8 & 9.5 & 7.8 & 7.2 & 3.9 & 3.7 & 3.5 & 3.1 & 3.0 & 2.9 & 2.8 & 2.6 & 2.4 & 1.8 & 1.6 & 1.2 & 0.8 & 0.6 & 0.6 & & 3.8 \\
\hline $\mathrm{N}$ & 8.7 & 6.5 & 5.3 & 4.9 & 2.6 & 2.5 & 2.4 & 2.1 & 2.0 & 1.9 & 1.9 & 1.8 & 1.6 & 1.2 & 1.1 & 0.8 & 0.6 & 0.4 & 0.4 & & 2.6 \\
\hline$S$ & 8.3 & 6.2 & 5.0 & 4.7 & 2.5 & 2.4 & 2.3 & 2.0 & 2.0 & 1.9 & 1.8 & 1.7 & 1.6 & 1.2 & 1.1 & 0.8 & 0.5 & 0.4 & 0.4 & & 2.5 \\
\hline $\mathrm{R}$ & 6.9 & 5.1 & 4.2 & 3.9 & 2.1 & 2.0 & 1.9 & 1.7 & 1.6 & 1.5 & 1.5 & 1.4 & 1.3 & 1.0 & 0.9 & 0.6 & 0.4 & 0.3 & 0.3 & & 2.0 \\
\hline$T$ & 6.6 & 4.9 & 4.0 & 3.7 & 2.0 & 1.9 & 1.8 & 1.6 & 1.6 & 1.5 & 1.4 & 1.4 & 1.2 & 0.9 & 0.9 & 0.6 & 0.4 & 0.3 & 0.3 & & 1.9 \\
\hline$Q$ & 6.6 & 4.9 & 4.0 & 3.7 & 2.0 & 1.9 & 1.8 & 1.6 & 1.6 & 1.5 & 1.4 & 1.4 & 1.2 & 0.9 & 0.9 & 0.6 & 0.4 & 0.3 & 0.3 & & 1.9 \\
\hline G & 6.2 & 4.6 & 3.7 & 3.5 & 1.9 & 1.8 & 1.7 & 1.5 & 1.4 & 1.4 & 1.3 & 1.3 & 1.2 & 0.9 & 0.8 & 0.6 & 0.4 & 0.3 & 0.3 & & 1.8 \\
\hline $\mathrm{K}$ & 5.5 & 4.1 & 3.3 & 3.1 & 1.7 & 1.6 & 1.5 & 1.3 & 1.3 & 1.2 & 1.2 & 1.1 & 1.0 & 0.8 & 0.7 & 0.5 & 0.3 & 0.3 & 0.2 & & 1.6 \\
\hline$M$ & 5.4 & 4.0 & 3.3 & 3.0 & 1.6 & 1.5 & 1.5 & 1.3 & 1.3 & 1.2 & 1.2 & 1.1 & 1.0 & 0.7 & 0.7 & 0.5 & 0.3 & 0.3 & 0.2 & & 1.6 \\
\hline $\mathrm{F}$ & 4.8 & 3.6 & 2.9 & 2.7 & 1.5 & 1.4 & 1.3 & 1.2 & 1.1 & 1.1 & 1.1 & 1.0 & 0.9 & 0.7 & 0.6 & 0.4 & 0.3 & 0.2 & 0.2 & & 1.4 \\
\hline$Y$ & 4.7 & 3.5 & 2.8 & 2.6 & 1.4 & 1.3 & 1.3 & 1.1 & 1.1 & 1.0 & 1.0 & 1.0 & 0.9 & 0.7 & 0.6 & 0.4 & 0.3 & 0.2 & 0.2 & & 1.4 \\
\hline A & 4.2 & 3.1 & 2.5 & 2.3 & 1.3 & 1.2 & 1.1 & 1.0 & 1.0 & 0.9 & 0.9 & 0.9 & 0.8 & 0.6 & 0.5 & 0.4 & 0.3 & 0.2 & 0.2 & & 1.2 \\
\hline W & 3.2 & 2.4 & 2.0 & 1.8 & 1.0 & 0.9 & 0.9 & 0.8 & 0.8 & 0.7 & 0.7 & 0.7 & 0.6 & 0.5 & 0.4 & 0.3 & 0.2 & 0.2 & 0.1 & & 1.0 \\
\hline V & 3.0 & 2.2 & 1.8 & 1.7 & 0.9 & 0.9 & 0.8 & 0.7 & 0.7 & 0.7 & 0.7 & 0.6 & 0.6 & 0.4 & 0.4 & 0.3 & 0.2 & 0.1 & 0.1 & & 0.9 \\
\hline$E$ & 3.0 & 2.2 & 1.8 & 1.7 & 0.9 & 0.9 & 0.8 & 0.7 & 0.7 & 0.7 & 0.7 & 0.6 & 0.6 & 0.4 & 0.4 & 0.3 & 0.2 & 0.1 & 0.1 & & 0.9 \\
\hline D & 2.8 & 2.1 & 1.7 & 1.6 & 0.8 & 0.8 & 0.8 & 0.7 & 0.7 & 0.6 & 0.6 & 0.6 & 0.5 & 0.4 & 0.4 & 0.3 & 0.2 & 0.1 & 0.1 & & 0.8 \\
\hline L & 2.6 & 1.9 & 1.6 & 1.4 & 0.8 & 0.7 & 0.7 & 0.6 & 0.6 & 0.6 & 0.6 & 0.5 & 0.5 & 0.4 & 0.3 & 0.2 & 0.2 & 0.1 & 0.1 & & 0.8 \\
\hline I & 2.5 & 1.8 & 1.5 & 1.4 & 0.7 & 0.7 & 0.7 & 0.6 & 0.6 & 0.5 & 0.5 & 0.5 & 0.5 & 0.3 & 0.3 & 0.2 & 0.2 & 0.1 & 0.1 & & 0.7 \\
\hline$P$ & 2.4 & 1.8 & 1.5 & 1.3 & 0.7 & 0.7 & 0.7 & 0.6 & 0.6 & 0.5 & 0.5 & 0.5 & 0.4 & 0.3 & 0.3 & 0.2 & 0.2 & 0.1 & 0.1 & & 0.7 \\
\hline Ave. & 5.7 & 4.3 & 3.5 & 3.2 & 1.7 & 1.6 & 1.6 & 1.4 & 1.3 & 1.3 & 1.3 & 1.2 & 1.1 & 0.8 & 0.7 & 0.5 & 0.4 & 0.3 & 0.3 & & 1.69 \\
\hline
\end{tabular}

a Nguyen, D., Mayne, L., Phillips, M.C., Englander, S.W.: Reference Parameters for Protein Hydrogen Exchange Rates. J. Amer. Soc. Mass Spectrom., 29, 1936-1939 (2018)

Table S3. Errors using polyalanine instead of actual peptide sequence in free energy $(\mathrm{kcal} / \mathrm{mol})$.

\begin{tabular}{|c|c|c|c|c|c|c|c|c|c|c|c|c|c|c|c|c|c|c|c|c|c|}
\hline $\mathrm{XIY}$ & C & $\mathrm{N}$ & $\mathrm{H}$ & $S$ & $\mathrm{D}$ & $\mathrm{R}$ & $\mathrm{Q}$ & A & $\mathrm{M}$ & $\mathrm{G}$ & $\mathrm{K}$ & $\mathrm{T}$ & $E$ & $\mathrm{~F}$ & $Y$ & $\mathrm{~W}$ & $\mathrm{~L}$ & V & 1 & $P$ & Ave. \\
\hline C & 1.6 & 1.4 & 1.3 & 1.2 & 0.9 & 0.8 & 0.8 & 0.7 & 0.7 & 0.7 & 0.7 & 0.7 & 0.6 & 0.4 & 0.4 & 0.2 & 0.0 & 0.2 & 0.2 & & 0.7 \\
\hline $\mathrm{H}$ & 1.5 & 1.3 & 1.2 & 1.2 & 0.8 & 0.8 & 0.7 & 0.7 & 0.6 & 0.6 & 0.6 & 0.6 & 0.5 & 0.3 & 0.3 & 0.1 & 0.1 & 0.3 & 0.3 & & 0.7 \\
\hline $\mathrm{N}$ & 1.3 & 1.1 & 1.0 & 0.9 & 0.6 & 0.5 & 0.5 & 0.4 & 0.4 & 0.4 & 0.4 & 0.3 & 0.3 & 0.1 & 0.1 & 0.1 & 0.3 & 0.5 & 0.6 & & 0.5 \\
\hline$S$ & 1.2 & 1.1 & 1.0 & 0.9 & 0.5 & 0.5 & 0.5 & 0.4 & 0.4 & 0.4 & 0.4 & 0.3 & 0.3 & 0.1 & 0.0 & 0.1 & 0.4 & 0.5 & 0.6 & & 0.5 \\
\hline $\mathrm{R}$ & 1.1 & 1.0 & 0.8 & 0.8 & 0.4 & 0.4 & 0.4 & 0.3 & 0.3 & 0.3 & 0.2 & 0.2 & 0.2 & 0.0 & 0.1 & 0.3 & 0.5 & 0.7 & 0.7 & & 0.5 \\
\hline$T$ & 1.1 & 0.9 & 0.8 & 0.8 & 0.4 & 0.4 & 0.4 & 0.3 & 0.3 & 0.2 & 0.2 & 0.2 & 0.1 & 0.0 & 0.1 & 0.3 & 0.5 & 0.7 & 0.7 & & 0.4 \\
\hline$Q$ & 1.1 & 0.9 & 0.8 & 0.8 & 0.4 & 0.4 & 0.4 & 0.3 & 0.3 & 0.2 & 0.2 & 0.2 & 0.1 & 0.0 & 0.1 & 0.3 & 0.5 & 0.7 & 0.7 & & 0.4 \\
\hline G & 1.1 & 0.9 & 0.8 & 0.7 & 0.4 & 0.3 & 0.3 & 0.2 & 0.2 & 0.2 & 0.2 & 0.1 & 0.1 & 0.1 & 0.1 & 0.3 & 0.6 & 0.7 & 0.8 & & 0.4 \\
\hline $\mathrm{K}$ & 1.0 & 0.8 & 0.7 & 0.7 & 0.3 & 0.3 & 0.2 & 0.2 & 0.2 & 0.1 & 0.1 & 0.1 & 0.0 & 0.2 & 0.2 & 0.4 & 0.6 & 0.8 & 0.8 & & 0.4 \\
\hline$M$ & 1.0 & 0.8 & 0.7 & 0.7 & 0.3 & 0.3 & 0.2 & 0.1 & 0.1 & 0.1 & 0.1 & 0.1 & 0.0 & 0.2 & 0.2 & 0.4 & 0.6 & 0.8 & 0.8 & & 0.4 \\
\hline$F$ & 0.9 & 0.7 & 0.6 & 0.6 & 0.2 & 0.2 & 0.2 & 0.1 & 0.1 & 0.0 & 0.0 & 0.0 & 0.1 & 0.2 & 0.3 & 0.5 & 0.7 & 0.9 & 0.9 & & 0.4 \\
\hline$Y$ & 0.9 & 0.7 & 0.6 & 0.6 & 0.2 & 0.2 & 0.1 & 0.1 & 0.1 & 0.0 & 0.0 & 0.0 & 0.1 & 0.3 & 0.3 & 0.5 & 0.7 & 0.9 & 0.9 & & 0.4 \\
\hline A & 0.8 & 0.7 & 0.5 & 0.5 & 0.1 & 0.1 & 0.1 & 0.0 & 0.0 & 0.0 & 0.1 & 0.1 & 0.1 & 0.3 & 0.4 & 0.6 & 0.8 & 1.0 & 1.0 & & 0.4 \\
\hline W & 0.7 & 0.5 & 0.4 & 0.4 & 0.0 & 0.0 & 0.1 & 0.1 & 0.2 & 0.2 & 0.2 & 0.2 & 0.3 & 0.5 & 0.5 & 0.7 & 0.9 & 1.1 & 1.1 & & 0.4 \\
\hline V & 0.7 & 0.5 & 0.4 & 0.3 & 0.1 & 0.1 & 0.1 & 0.2 & 0.2 & 0.2 & 0.2 & 0.3 & 0.3 & 0.5 & 0.6 & 0.7 & 1.0 & 1.1 & 1.2 & & 0.5 \\
\hline$E$ & 0.6 & 0.5 & 0.3 & 0.3 & 0.1 & 0.1 & 0.1 & 0.2 & 0.2 & 0.2 & 0.3 & 0.3 & 0.3 & 0.5 & 0.6 & 0.8 & 1.0 & 1.1 & 1.2 & & 0.5 \\
\hline D & 0.6 & 0.4 & 0.3 & 0.3 & 0.1 & 0.1 & 0.2 & 0.2 & 0.3 & 0.3 & 0.3 & 0.3 & 0.4 & 0.6 & 0.6 & 0.8 & 1.0 & 1.2 & 1.2 & & 0.5 \\
\hline L & 0.6 & 0.4 & 0.3 & 0.2 & 0.1 & 0.2 & 0.2 & 0.3 & 0.3 & 0.3 & 0.3 & 0.4 & 0.4 & 0.6 & 0.7 & 0.8 & 1.1 & 1.2 & 1.3 & & 0.5 \\
\hline I & 0.5 & 0.4 & 0.2 & 0.2 & 0.2 & 0.2 & 0.2 & 0.3 & 0.3 & 0.4 & 0.4 & 0.4 & 0.5 & 0.6 & 0.7 & 0.9 & 1.1 & 1.3 & 1.3 & & 0.5 \\
\hline $\mathrm{P}$ & 0.5 & 0.3 & 0.2 & 0.2 & 0.2 & 0.2 & 0.2 & 0.3 & 0.3 & 0.4 & 0.4 & 0.4 & 0.5 & 0.6 & 0.7 & 0.9 & 1.1 & 1.3 & 1.3 & & 0.5 \\
\hline Ave. & 0.9 & 0.8 & 0.6 & 0.6 & 0.3 & 0.3 & 0.3 & 0.3 & 0.3 & 0.3 & 0.3 & 0.3 & 0.3 & 0.3 & 0.3 & 0.5 & 0.7 & 0.8 & 0.9 & & 0.47 \\
\hline
\end{tabular}

value in $\mathrm{S} 3=\mid R T \ln$ (value in $\mathrm{S} 2$ ) $\mid$ with $R=0.001987$ and $T=296$. 


\section{Derivations to Calculate Area between Two Exponential Curves}

\subsection{Calculation of Area between Two Exponential Curves by Frullani integral}

$$
\begin{aligned}
& D^{-}{ }_{\text {obs }}(x)=1-\exp \left(-k^{-}{ }_{\text {obs }} 10^{x}\right) \\
& D^{+}{ }_{\text {obs }}(x)=1-\exp \left(-k^{+}{ }_{\text {obs }} 10^{x}\right)
\end{aligned}
$$

The area between Eqs. 8a and $8 \mathrm{~b}$ is defined as Eq. S1.

$$
\begin{aligned}
\Delta A_{\mathrm{obs}} & =\int_{-\infty}^{\infty}\left[D^{-}{ }_{\text {obs }}(x)-D^{+}{ }_{\text {obs }}(x)\right] \mathrm{d} x \\
& =\int_{-\infty}^{\infty}\left\{\left[1-\exp \left(-k_{\text {obs }}^{-} 10^{x}\right)\right]-\left[1-\exp \left(-k^{+}{ }_{\text {obs }} 10^{x}\right)\right]\right\} \mathrm{d} x \\
& =\int_{-\infty}^{\infty}\left[\exp \left(-k^{+}{ }_{\text {obs }} 10^{x}\right)-\exp \left(-k^{-}{ }_{\text {obs }} 10^{x}\right)\right] \mathrm{d} x
\end{aligned}
$$

Assuming $t=10^{x}$, Eq. S1 becomes Eq. S2.

$$
\begin{aligned}
\Delta A_{\mathrm{obs}} & =\int_{0}^{\infty}\left[\exp \left(-k^{+}{ }_{\mathrm{obs}} t\right)-\exp \left(-k_{\mathrm{obs}}^{-} t\right)\right] \frac{\mathrm{d} x}{\mathrm{~d} t} \mathrm{~d} t \\
& =\int_{0}^{\infty}\left[\exp \left(-k_{\mathrm{obs}}^{+} t\right)-\exp \left(-k_{\mathrm{obs}} t\right)\right] \frac{1}{t \ln 10} \mathrm{~d} t \\
& =\frac{1}{\ln 10} \int_{0}^{\infty} \frac{\exp \left(-k_{\mathrm{obs}}^{+} t\right)-\exp \left(-k_{\mathrm{obs}}^{-} t\right)}{t} \mathrm{~d} t
\end{aligned}
$$

Applying Frullani integral with $f(x)=\exp (-x)$, Eq. S2 can be solved as

$$
\begin{aligned}
\Delta A_{\mathrm{obs}} & =\frac{1}{\ln 10}\left[(\exp (-\infty)-\exp (0)] \ln \frac{k_{\mathrm{obs}}^{+}}{k_{\mathrm{obs}}^{-}}\right. \\
& =\frac{1}{\ln 10}(-1) \ln \frac{k_{\mathrm{obs}}^{+}}{k_{\mathrm{obs}}^{-}} \\
& =\frac{1}{\ln 10} \ln \frac{k_{\mathrm{obs}}^{-}}{k_{\mathrm{obs}}^{+}} \\
& =\log _{10} \frac{k_{\mathrm{obs}}}{k_{\mathrm{obs}}^{+}}
\end{aligned}
$$

\subsection{Calculation of Area between Two Stretched Exponential Curves}


The definition of stretched exponential curve is from main text,

$$
y=D \%_{\mathrm{SE}}(x)=1-\exp \left[-\left(k_{\mathrm{SE}} 10^{x}\right)^{B}\right]
$$

where $k_{\mathrm{SE}}$ is rate factor which becomes larger when HDX rates in the segment is faster and $B$ is stretch factor which becomes larger when the HDX curve is steeper. Eq. 19a becomes

$$
\begin{aligned}
& y=1-\exp \left[-\left(k_{\mathrm{SE}} 10^{x}\right)^{B}\right] \\
& \exp \left[-\left(k_{\mathrm{SE}} 10^{x}\right)^{B}\right]=1-y \\
& -\left(k_{\mathrm{SE}} 10^{x}\right)^{B}=\ln (1-y) \\
& \left(k_{\mathrm{SE}} 10^{x}\right)^{B}=-\ln (1-y) \\
& k_{\mathrm{SE}} 10^{x}=[-\ln (1-y)]^{1 / B} \\
& 10^{x}=\left\{[-\ln (1-y)]^{1 / B}\right\} / k_{\mathrm{SE}} \\
& x=\log _{10}\left\{[-\ln (1-y)]^{1 / B} / k_{\mathrm{SE}}\right\} \\
& x=\log _{10}[-\ln (1-y)]^{1 / B}-\log _{10} k_{\mathrm{SE}} \\
& x=(1 / B) \log _{10}[-\ln (1-y)]-\log _{10} k_{\mathrm{SE}} \\
& x=[1 /(B \ln 10)] \ln [-\ln (1-y)]-\log _{10} k_{\mathrm{SE}}
\end{aligned}
$$

When HDX-MS data with $(+)$ and without $(-)$ perturbation are acquired, the two fitted equations are

$$
\begin{aligned}
& D \%_{\mathrm{SE}^{-}}(x)=1-\exp \left[-\left(k_{\mathrm{SE}^{-}} 10^{x}\right)^{B-}\right] \\
& D \%_{\mathrm{SE}^{+}}(x)=1-\exp \left[-\left(k_{\mathrm{SE}^{+}} 10^{x}\right)^{B+}\right]
\end{aligned}
$$

Let us calculate the area between two stretched exponential curves.

Eqs. $19 \mathrm{~b}$ and 19c become Eqs. S3b and S3c in the same way as Eq. 19a became Eq. S3a.

$$
\begin{aligned}
& x_{-}=\left[1 /\left(B_{-} \ln 10\right)\right] \ln [-\ln (1-y)]-\log _{10} k_{\mathrm{SE}^{-}} \\
& x_{+}=\left[1 /\left(B_{-} \ln 10\right)\right] \ln [-\ln (1-y)]-\log _{10} k_{\mathrm{SE}^{+}}
\end{aligned}
$$


At a given $y$, the distance in $x$-dimension, $\Delta x$, is

$$
\begin{aligned}
\Delta x & =\left[1 /\left(B^{+} \ln 10\right)\right] \ln [-\ln (1-y)]-\log _{10} k^{+}{ }_{\mathrm{SE}}-\left(1 / B_{-} \ln 10\right) \ln [-\ln (1-y)]+\log _{10} k_{\mathrm{SE}} \\
& =\left(1 / B^{+}-1 / B^{-}\right)(1 / \ln 10) \ln [-\ln (1-y)]-\log _{10} k_{\mathrm{SE}}^{+}+\log _{10} k_{\mathrm{SE}}^{-} \quad \text { (Eq. S4) }
\end{aligned}
$$

Integration of $\Delta x$ between $y=0$ and 1 gives the area between the two stretched exponential curves

$$
\Delta A \%_{\mathrm{SE}}=\left(1 / B^{+}-1 / B^{-}\right)(1 / \ln 10) \int_{0}^{1} \ln [-\ln (1-y)] \mathrm{d} y-\log _{10} k^{+}{ }_{\mathrm{SE}}+\log _{10} k_{\mathrm{SE}}^{-}
$$

(Eq. S5)

Since $\left(1 / B^{+}-1 / B^{-}\right)(1 / \ln 10)$ and $-\log _{10} k^{+}{ }_{\mathrm{SE}}+\log _{10} k_{\mathrm{SE}}$ are constant, $\Delta A_{\mathrm{I}}$ needs to be solved to obtain $\Delta A_{\mathrm{SE}}$.

$$
\Delta A_{\mathrm{I}}=\int_{0}^{1} \ln [-\ln (1-y)] \mathrm{d} y
$$

Asume $z=1-y$,

$$
\Delta A_{\mathrm{I}}=-\int_{1}^{0} \ln [\ln (1 / z)] \mathrm{d} z
$$

Asume $w=1 / z$,

$$
\Delta A_{\mathrm{I}}=\int_{1}^{\infty} \ln [\ln w]\left(1 / w^{2}\right) \mathrm{d} w
$$

Asume $t=\ln w\left(w=\mathrm{e}^{t}\right)$,

$$
\Delta A_{\mathrm{I}}=\int_{0}^{\infty} \ln [t]\left(\mathrm{e}^{-t}\right) \mathrm{d} t
$$

Solving Eq. S9 (p.2416 of CRC Handbook of Chemistry and Physicis $84^{\text {th }}$ Edition) yields,

$$
\Delta A_{\mathrm{I}}=-\gamma(=0.5772156649, \text { Euler-Mascheroni constant })
$$

Using Eq. S10, Eq. S5 became,

$$
\begin{aligned}
& \Delta A \%_{\mathrm{SE}}=-(\gamma / \ln 10)\left(1 / B^{+}-1 / B^{-}\right)-\log _{10} k^{+}{ }_{\mathrm{SE}}+\log _{10} k_{\mathrm{SE}}^{-} \\
& -\ln 10 R T \Delta A \%_{\mathrm{obs}}=\frac{1}{n} \sum_{i=1}^{n} \Delta G_{i}
\end{aligned}
$$

(Eq. 13b) 
Replacing $\Delta A \%_{\text {obs }}$ with $\Delta A \%_{\text {SE }}$ in Eq. 10 a,

$$
\begin{aligned}
\frac{1}{n} \sum_{i=1}^{n} \Delta G_{i} & =-\ln 10 R T\left[-(\gamma / \ln 10)\left(1 / B^{+}-1 / B^{-}\right)-\log _{10} k^{+}{ }_{\mathrm{SE}}+\log _{10} k_{\mathrm{SE}}\right] \\
& =R T\left[\gamma\left(1 / B^{+}-1 / B^{-}\right)+\ln {k^{+}}_{\mathrm{SE}}-\ln {k^{-}}_{\mathrm{SE}}\right]
\end{aligned}
$$

\subsection{Calculation of Protection Factor between Two Stretched Exponential Curves}

$$
\begin{gathered}
\Delta G=-R T \ln p f \\
\frac{1}{n} \sum_{i=1}^{n} \Delta G_{i}=\Delta G_{\mathrm{ave}}=-R T \ln p f_{\mathrm{ave}}
\end{gathered}
$$

Combining Eq. S12 with Eq. S13,

$$
\begin{aligned}
& -R T \ln p f_{\mathrm{are}}=R T\left[\gamma\left(1 / B_{+}-1 / B^{-}\right)+\ln k^{+}{ }_{\mathrm{SE}}-\ln k_{\mathrm{SE}}\right] \\
& -\ln p f_{\mathrm{are}}=\gamma\left(1 / B^{+}-1 / B^{-}\right)+\ln k_{\mathrm{SE}}^{+}-\ln k_{\mathrm{SE}} \\
& \ln k_{\mathrm{SE}}^{-}-\ln k_{\mathrm{SE}}^{+}-\ln p f_{\mathrm{are}}=\gamma\left(1 / B^{+}-1 / B^{-}\right) \\
& \ln \left[k_{\mathrm{SE}}^{-} /\left(k_{\mathrm{SE}}^{+} p f_{\mathrm{are}}\right)\right]=\gamma\left(1 / B^{+}-1 / B^{-}\right) \\
& k_{\mathrm{SE}}^{-} /\left(k_{\mathrm{SE}}^{+} p f_{\mathrm{are}}\right)=e^{\gamma\left(1 / B^{+-1 / B-}\right)} \\
& p f_{\mathrm{are}}=\frac{k_{\mathrm{SE}}^{-}\left(e^{\gamma}\right)^{1 / B_{-}}}{k_{\mathrm{SE}}^{+}\left(e^{\gamma}\right)^{1 / B^{+}}} \\
& p f_{\mathrm{are}}=\frac{k_{\mathrm{SE}}^{-} 1.78^{1 / B-}}{k_{\mathrm{SE}}^{+} 1.78^{1 / B^{+}}}
\end{aligned}
$$

